\title{
Genetic Strain-Dependent Protein Metabolism and Muscle Hypertrophy Under Chronic Isometric Training in Rat Gastrocnemius Muscle
}

\author{
K. KOBAYASHI ${ }^{1}$, R. OGASAWARA ${ }^{2}$, A. TSUTAKI ${ }^{1}$, K. LEE $^{1}$, E. OCHI ${ }^{3}$, K. NAKAZATO ${ }^{1}$ \\ ${ }^{1}$ Graduate School of Health and Sport Sciences, Nippon Sport Science University, Tokyo, Japan, \\ ${ }^{2}$ Department of Human and Engineered Environmental Studies, Graduate School of Frontier \\ Sciences, The University of Tokyo, Kashiwanoha, Kashiwa, Chiba, Japan, ${ }^{3}$ Laboratory of Health \\ and Sports Sciences, Center for Liberal Arts, Meiji Gakuin University, Yokohama, Kanagawa, \\ Japan
}

Received December 27, 2011

Accepted June 1, 2012

On-line August 8, 2012

\section{Summary}

Genetic strain-dependent reactivity to mechanical stimuli in rat skeletal muscle has not been examined. This study aimed to examine whether genetic strain-dependency is associated with reactivity in protein metabolism and the resultant muscle hypertrophy after isometric resistance training (RT). The right triceps of Sprague-Dawley (SD) and Wistar rats underwent 12 sessions of RT. After RT, a transition from the IIb to the IIx myosin heavy-chain isoform was observed in both strains. In SD rats, the lateral gastrocnemius muscle (LG) mass of the trained legs (TRN) was significantly higher than that of the control legs (CON) $(7.8 \%, \mathrm{P}<0.05)$. Meanwhile, in Wistar rats, the LG mass was unchanged. In SD rats, the levels of 70-kDa ribosomal protein S6 kinase (p70S6k) and forkhead box 3a (FOXO3a) phosphorylation in the TRN were significantly greater than those of the CON (2.2- and 1.9-fold, respectively; $P<0.05)$. The expression of muscle ring finger-1 (MuRF1) and muscle atrophy F-box (MAFbx/atrogin-1) in the TRN were significantly lower than those of the CON (0.6- and 0.7-fold, respectively; $\mathrm{P}<0.05$ ). However, in Wistar rats, there was no significant difference. These results suggest a genetic strain difference in protein metabolism. This phenomenon may be useful for studying individual differences in response to RT.

\section{Key words}

Rat strains • Muscle hypertrophy • Resistance training • Protein synthesis • Protein degradation

\section{Corresponding author}

Koji Kobayashi, 7-1-1, Fukasawa, Setagaya-ku, Tokyo 158-8508, Japan. E-mail: kobakoji0518@yahoo.co.jp

\section{Introduction}

Skeletal muscle size is believed to be regulated by both muscle protein synthesis (MPS) and muscle protein breakdown (MPB). Appropriate stimulation of skeletal muscle enhances MPS, resulting in muscle hypertrophy. A number of studies have confirmed this (Dreyer et al. 2006, Dreyer et al. 2008). Conversely, unloading (i.e., hindlimb unloading (Hornberger et al. 2001, Haddad et al. 2006) and denervation (Hornberger et al. 2001)) induce muscle atrophy as a result of increased MPB-related indexes.

Anabolic reactions in skeletal muscle exerted by resistance exercise (Biolo et al. 1995), electrical stimulation (Baar and Esser 1999, Nader and Esser 2001), and compensatory overload (Bodine et al. 2001) have been investigated at the molecular level. For example, it was reported that acute bouts of eccentric knee extension augmented the phosphorylation of Akt, mammalian target of rapamycin (mTOR), and 70-kDa ribosomal protein S6 kinase (p70S6k); these are translation regulator phosphorylation proteins belonging to the serine/threonine kinases, which are related to protein synthesis (Roschel et al. 2011). In agreement with these 
findings, resistance training (RT) resulting in muscle hypertrophy is closely involved in the activities of the Akt/mTOR/p70S6k pathway (Ochi et al. 2010), leading to an anabolic response.

Protein synthesis and protein degradation following resistance exercise have recently been investigated at the molecular level. Louis et al. (2007) clarified the impact of acute exercise on muscle ring finger 1 (MuRF1) and muscle atrophy F-box (MAFbx/atrogin-1), which are ubiquitin ligases (E3) involved in muscular protein degradation (Sandri et al. 2004, Stitt et al. 2004). E3 is regulated by Forkhead transcription factor (FOXO). In situations where protein synthesis was increased, FOXO was phosphorylated by Akt and was localized in the cytosol. When FOXO is dephosphorylated, it is transported from the cytosol to the nucleus, leading to higher expression of E3 expression (Sandri et al. 2004). It has been confirmed that RT leading to skeletal muscle hypertrophy decreases MuRF1 and MaFbx/Atrogin-1 mRNA expression in rats (Zanchi et al. 2009).

Since Adams et al. (2004) and Haddad et al. (1998) report that all 3 contraction modes (i.e., isometric, concentric, and eccentric contraction) successfully induce muscle hypertrophy in Sprague-Dawley (SD) rat Gastrocnemius (GA), we also applied isometric RT to Wistar rat GA to further elucidate the molecular mechanisms of muscle hypertrophy. Contrary to expectations, we were unable to confirm muscle hypertrophy (our unpublished observation). Such straindependent reactions suggest that anabolic and/or catabolic responses should differ between Wistar and SD rats. In fact, Soukup and his colleagues have reported inter-strain differences are present in muscle fiber type composition (Novák et al. 2010). They found that the proportion of fast fibers in the soleus is larger in Spontaneously Hypertensive (SHR) rat than in Wistar rats. This difference is in close agreement with differences in twitch contraction and relaxation time (Lewis et al. 1994). As skeletal muscle hypertrophy occurs mainly in fast fibers, the lower content of fast fibers in Wistar rat skeletal muscle might decrease the hypertrophic response to resistance training. Inter-strain differences in endocrine secretion may also have influenced the muscle hypertrophy results.

This study aimed to elucidate the molecular background underlying the genetic strain-dependent response of rats to chronic isometric training. We focused on protein synthesis and degradation reactions in both SD and Wistar rats to examine the detailed differences between these strains.

\section{Materials and Methods}

Animals

Male SD rats (age, 10 weeks; body mass, 300$330 \mathrm{~g} ; \mathrm{n}=5$ ) (CLEA Japan, Tokyo, Japan) and male Wistar rats (age, 10 weeks; body mass, 290-320 g; n=6) (CLEA Japan, Tokyo, Japan) were used in this study. Each strain underwent RT: 12 sessions of isometric RT in the SD $(n=5)$ and Wistar rat groups $(n=6)$. The right GA was trained, and the left GA was used as a control. Thus, we formed 4 groups as follows: the trained SD group (SD-TRN), control SD group (SD-CON), trained Wistar group (W-TRN), and control Wistar group (W-CON).

The rats were housed in individually ventilated cage systems (Tecniplast, Milan, Italy) maintained at 22$24{ }^{\circ} \mathrm{C}$ with a 12:12-h light/dark cycle. This study was approved by the Ethical Committee of the Nippon Sports Science University on the Use of Animal Subject in Research (010-A04).

\section{Muscle activation and training apparatus}

For each training bout, the rats were lightly anesthetized with isoflurane (aspiration rate, $450 \mathrm{ml} / \mathrm{min}$; concentration, $2.0 \%$ ), and the right hindlimbs were shaved. Stimulation electrodes coated with urethane wire (Unique Medical, Tokyo, Japan) were introduced subcutaneously in the region adjacent to the popliteal fossa via 27-gauge hypodermic needles that were subsequently withdrawn, leaving the electrode in place. Before insertion, a section of the urethane was removed, leaving the wire exposed in the area lateral and medial to the sciatic nerve and to permit field stimulation of the nerve. The electrodes were connected to an electric stimulator and isolator (SS-104; Nihon Koden, Tokyo, Japan).

When the stimulation electrodes were in place, the rats were positioned on the platform of a training dynamometer. The right leg was then positioned in a footplate attached to the dynamometer. The stimulation intensity was adjusted to produce maximal isometric tension (pulse duration: $0.4 \mathrm{~ms}$; frequency: $60 \mathrm{~Hz}$; intensity, $\sim 19 \mathrm{~V})$.

\section{Training protocol}

The model used in the present study is similar to that described by Haddad et al. (1998) with some 
modifications. The triceps surae muscle in each TRN was stimulated 5 times for isometric contraction ( $5 \mathrm{~s}$ ) with $5 \mathrm{~s}$ of rest time between contractions; each training session consisted of 5 sets with a rest interval of 5 min between each set. The rats were positioned with the right foot on the footplate at $80^{\circ}$ relative to the tibia. After each training session, the electrodes were withdrawn. The training regimen involved a sequence of 2 days of training followed by a day of rest. Twenty-four hour after the last exercise session, the GA muscle was dissected, ground to a powder, and immediately frozen in liquid $\mathrm{N}_{2}$ and stored at $-80^{\circ} \mathrm{C}$ until analysis. The GA of the trained right-leg was compared with that of the contralateral (left) leg. Posterior legs muscles are made up of GA (medial and lateral), soleus and plantaris. Both of SD and Wistar rats, lateral GA (LG) account for the greatest proportion (about $40 \%$ ) of posterior legs. Thus we believe that the changes seen in the LG reflect a response to stimulation used in this study. Therefore LG was used for further biochemical analysis.

\section{Myosin heavy-chain (MyHC) isoform analysis}

Powdered muscles were homogenized in a sodium dodecyl sulphate (SDS) solution containing $10 \%$ w/v SDS, $40 \mathrm{mM}$ dithiothreitol (DTT), $5 \mathrm{nM}$ EDTA, and 0.1 M Tris-HCL buffer ( $\mathrm{pH} 8.0$ ) to give a final concentration of muscle tissue of $0.25 \mathrm{mg} / \mu \mathrm{l}$. We added Protease Inhibitor Cocktail for Use with Mammalian Cell and Tissue Extracts (Nacalai Tesque) to some sample homogenates at 1:100. These sample homogenates were heated at $85^{\circ} \mathrm{C}$ for $10 \mathrm{~min}$. The samples were diluted in $2 \times$ sample buffer $(1.0 \% \mathrm{v} / \mathrm{v} \beta$-mercaptoethanol $(\beta-\mathrm{ME})$ or $100 \mathrm{mM}$ DTT, $4.0 \% \mathrm{w} / \mathrm{v}$ SDS, $0.16 \mathrm{M}$ Tris- $\mathrm{HCl}$ (pH 6.8), $43 \%$ v/v glycerol, and $0.2 \% \mathrm{w} / \mathrm{v}$ bromophenol blue) and $\mathrm{dH}_{2} \mathrm{O}$ to give final protein concentrations of 10 $1280 \mathrm{ng} / \mu \mathrm{l}$ in $1 \times$ sample buffer.

Gel and transfer conditions as well as the method for detecting bands were performed as described previously (Mizunoya et al. 2008). The bands were quantified by densitometry using Light Capture (ATTO, Tokyo, Japan).

\section{Western blot analysis}

A $30 \mu \mathrm{g}$ total protein extract from the homogenized samples (as identified above) was mixed with sample buffer, boiled on SDS-polyacrylamide gel (10-12.5\%), and electrophoresed at $20 \mathrm{~mA}$. The samples were electrophoretically separated, and the separated proteins were then transferred onto polyvinylidene difluoride (PVDF) membranes (ATTO, Tokyo, Japan). The membranes were blocked with PBS containing $1 \%$ skimmed milk for $1 \mathrm{~h}$ and then incubated overnight at $4{ }^{\circ} \mathrm{C}$ with the following primary antibodies (all diluted 1:1,000): monoclonal anti-Akt (no. 2920; Cell Signaling Technology, Danvers, MA), monoclonal anti-Akt (P) (no. 4051; Cell Signaling Technology), monoclonal antimTOR (no. 2983; Cell Signaling Technology), polyclonal anti-mTOR (P) (no. 2971; Cell Signaling Technology), polyclonal anti-p70S6k (no. 9202; Cell Signaling Technology), polyclonal anti-p70S6k (P) (no. 9205; Cell Signaling Technology), monoclonal anti-FOXO1 (no. 2880; Cell Signaling Technology), polyclonal antiFOXO1 (P) (no. 9461; Cell Signaling Technology), monoclonal anti-FOXO3 (no. 2497; Cell Signaling Technology), polyclonal anti-FOXO3 (P) (no. 9466; Cell Signaling Technology), monoclonal anti- $\beta$ actin (no. 3700; Cell Signaling Technology), polyclonal antiMuRF1 (no. sc-32920; Santa Cruz Biotechnology, Santa Cruz, CA), and polyclonal anti-MaFbx/Atrogin-1 (no. sc33782; Santa Cruz Biotechnology). The membranes were then washed ( $5 \min \times 3$ times) and incubated overnight with the secondary antibody at $4{ }^{\circ} \mathrm{C}$. Horseradish peroxidase (HRP)-conjugated goat anti-mouse immunoglobulin $\mathrm{G}$ (IgG) or ant-rabbit IgG (dilution, $1: 10,000)$ was used as the secondary antibody (SuperSignal West Dura; Pierce Protein Research Products, Rockford, IL). Chemiluminescent signals were detected using a chemiluminescence detector (AE6961; ATTO) and quantified using a personal computer with image analysis software (SC Analyzer; ATTO). The band densities were expressed relative to those obtained for the control.

\section{Statistical analysis}

The Wilcoxon-signed-rank test was used to test for differences between TRN and CON. Furthermore, the Mann-Whitney U test was used to test for differences between SD and Wistar rats; the tested variables were body mass, each muscle mass, each muscle mass relative to body mass, and the MyHC isoform composition ratio. The level of significance was set at $\mathrm{P}<0.05$. All values are expressed as means \pm SE. All analyses were performed using SPSS for Windows (SPSS Japan, Tokyo, Japan). 
Table 1. Rat characteristics.

\begin{tabular}{|c|c|c|c|c|}
\hline & \multicolumn{2}{|c|}{ SD-IT } & \multicolumn{2}{|c|}{ W-IT } \\
\hline & $\mathrm{CON}$ & TRN & $\mathrm{CON}$ & TRN \\
\hline Body weight after the training ( $g$ ) & \multicolumn{2}{|c|}{$424.10 \pm 9.60^{\dagger}$} & \multicolumn{2}{|c|}{$340.18 \pm 4.34$} \\
\hline Medial gastrocnemius (mg) & $971.36 \pm 24.16^{\$ \$}$ & $1056.24 \pm 37.03 * \$ \$$ & $750.08 \pm 17.97$ & $777.07 \pm 11.45$ \\
\hline $\begin{array}{l}\text { Medial gastrocnemius / } \\
\text { Body weight }(\mathrm{mg} / \mathrm{g})\end{array}$ & $2.29 \pm 0.04$ & $2.49 \pm 0.07^{*}$ & $2.21 \pm 0.08$ & $2.26 \pm 0.05$ \\
\hline Lateral gastrocnemius (mg) & $1146.96 \pm 31.19$ & $1239.80 \pm 21.23 * \$ \$$ & $908.03 \pm 22.76$ & $878.63 \pm 38.13$ \\
\hline $\begin{array}{l}\text { Lateral gastrocnemius / } \\
\text { Body weight }(\mathrm{mg} / \mathrm{g})\end{array}$ & $2.70 \pm 0.05$ & $2.93 \pm 0.07^{*}$ & $2.67 \pm 0.05$ & $2.58 \pm 0.12$ \\
\hline Gastrocnemius (mg) & $2122.62 \pm 59.16^{\$ \$}$ & $2304.10 \pm 60.49 * \$ \$$ & $1658.25 \pm 33.72$ & $1655.72 \pm 53.44$ \\
\hline $\begin{array}{l}\text { Gastrocnemius / } \\
\text { Body weight (mg/g) }\end{array}$ & $5.01 \pm 0.10$ & $5.44 \pm 0.15^{* \$}$ & $4.87 \pm 0.07$ & $4.87 \pm 0.16$ \\
\hline
\end{tabular}

Values are means \pm SE. TRN, trained legs; CON, control legs; W, Wistar; IT, Isometric resistance training. ${ }^{\dagger} \mathrm{P}<0.01, \mathrm{SD}-\mathrm{IT}$ vs. W-IT, ${ }^{*} \mathrm{P}<0.05$, TRN vs. CON in SD-IT. ${ }^{\$} \mathrm{P}<0.05,{ }^{\$} \mathrm{P}<0.01, \mathrm{SD}$-IT vs. W-IT of CON or SD-IT vs. W-IT of TRN.

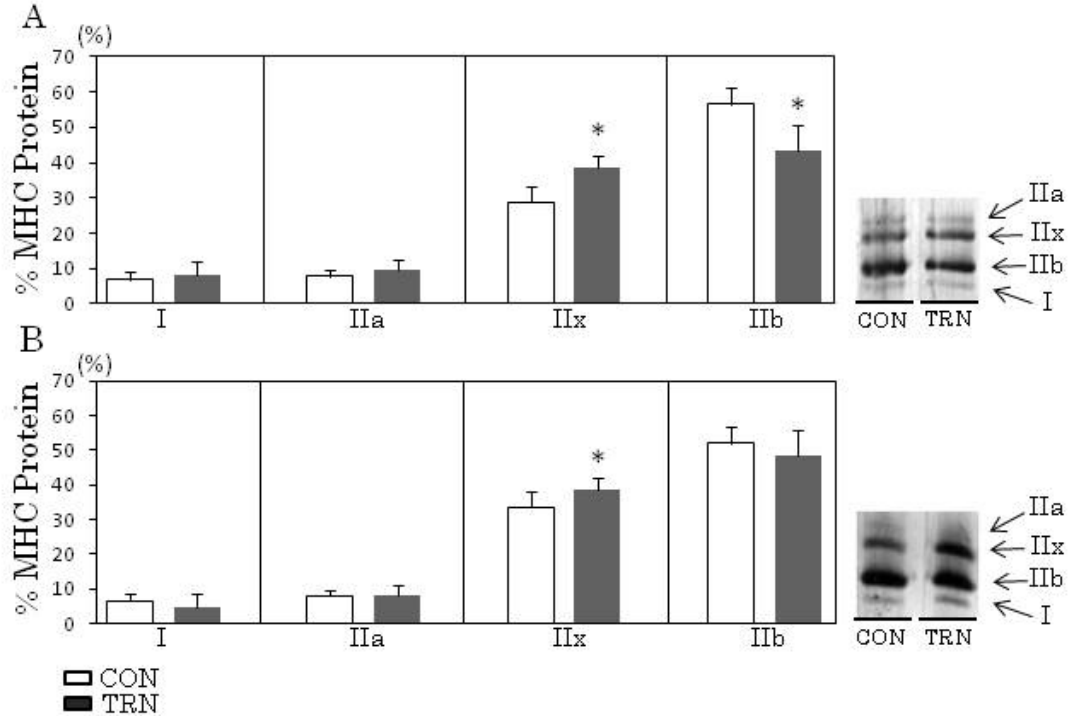

Fig. 1. Myosin heavy-chain (MyHC) isoform protein expression in the lateral gastrocnemius muscle (LG) after the isometric RT program. SD-IT (A). W-IT (B). Values are means $\pm \mathrm{SE}$ bars. ${ }^{*} \mathrm{P}<0.05$, TRN vs. CON. In the SD rats, the proportion of the IIx isoform was significantly higher in the TRN than in the CON [9.8\%; P<0.05 (A)]. The proportion of the IIb isoform in the TRN was significantly lower than in the CON $(13.1 \% ; \mathrm{P}<0.05)$. In the Wistar rats, the proportion of the IIx isoform in the TRN was significantly higher than that in the CON [4.91 \%; $\mathrm{P}<0.05$ (B)]. There were no significant differences between the SD-IT and W-IT groups.

\section{Results}

Body weights of rats and wet masses of GA muscles

The rat characteristics are presented in Table 1. The mean body weight, medial GA (MG) and GA masses of the CON, and MG, LG, and GA masses of the TRN of the $\mathrm{SD}$ rats were significantly greater than those of the Wistar rats $(\mathrm{P}<0.01)$. In addition, the GA mass of the TRN relative to the body mass of the $\mathrm{SD}$ rats was significantly greater than that of the Wistar rats $(\mathrm{P}<0.05)$.

In $\mathrm{SD}$ rats, the $\mathrm{MG}, \mathrm{LG}$, and GA masses and muscle mass/body weight ratios of the TRN were significantly higher than those of the CON $(\mathrm{P}<0.05)$. In contrast, in the Wistar rats, no significant difference was observed between the TRN and CON.

\section{Changes in MyHC isoform composition}

The MyHC isoforms were separated by electrophoresis and the proportions of the isoforms quantified by densitometry using Light Capture (ATTO, Tokyo, Japan).

In the SD rats, the proportion of the IIx isoform 


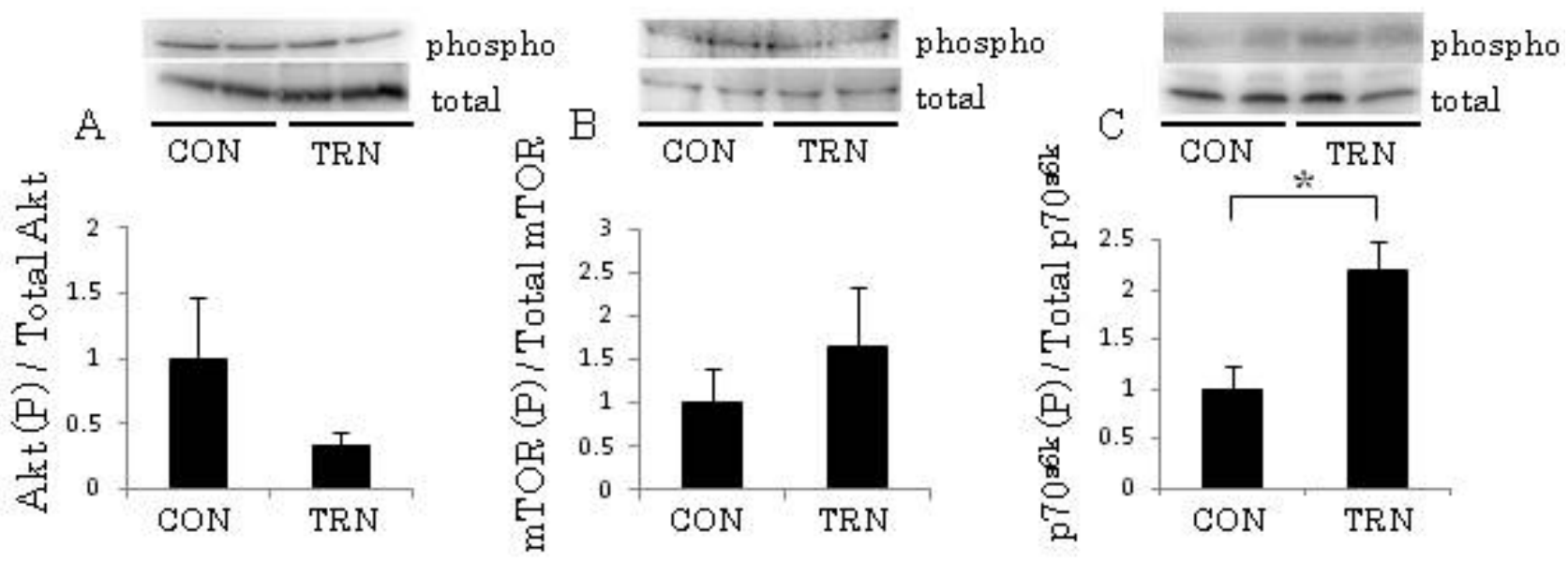

Fig. 2. The ratio of phosphorylated Akt $[A k t(P)]$ to total Akt $(\mathbf{A})$, the ratio of phosphorylated mammalian target of rapamycin [mTOR $(P)]$ to total mTOR (B), and the ratio of 70-kDa ribosomal protein S6 kinase [p70S6k (P)] to total p70S6k (C) in SD-TRN and -CON determined by western blotting. The ratio of p70S6k $(P)$ to total protein in the TRN was significantly greater than that in the CON (C). However, ratio of $A k t(P)$ to total Akt and mTOR(P) to total mTOR did not differ between the TRN and CON (A and B). Values are expressed as means $\pm \mathrm{SE}$. ${ }^{*} \mathrm{P}<0.05$, TRN vs. CON.
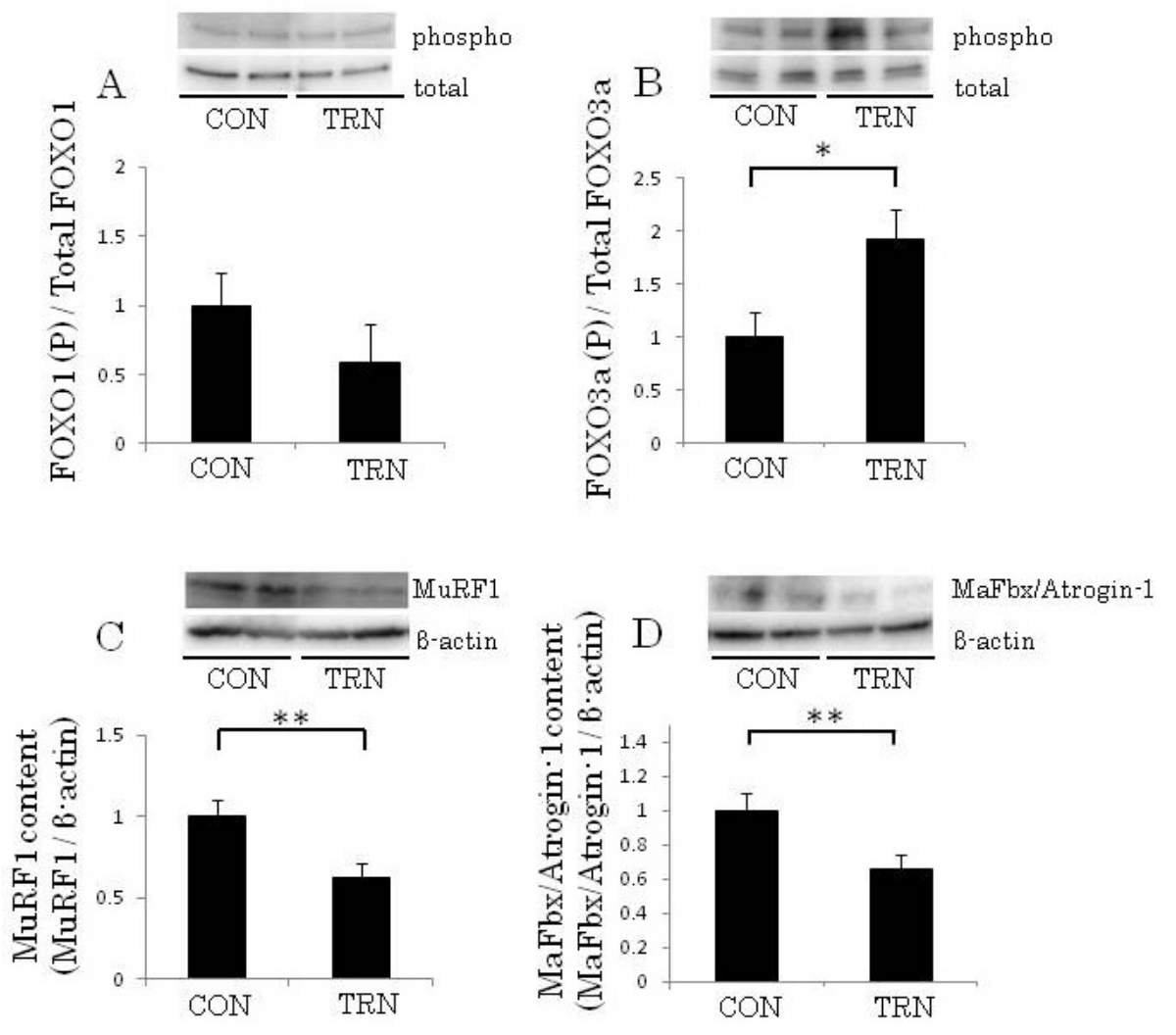

Fig. 3. The ratio of phosphorylated Forkhead box protein O1 [FOXO1 $(P)]$ to total protein $(\mathbf{A})$, the ratio of phosphorylated FOXO3a [FOXO3a $(\mathrm{P})$ ] to total protein (B), and the levels of MuRF1 (C) and MaFbx/Atrogin-1 (D) in SD-TRN and -CON determined by western blotting. The ratio of FOXO3a (P) to total FOXO3 in TRN was significantly greater than that in CON (B), whereas that of FOXO1 (A) did not differ significantly between TRN and CON. The muscle contents of MuRF1 and MaFbx/Atrogin-1 were significantly lower in TRN than in CON (C and D). Values are expressed as means $\pm \mathrm{SE}$. ${ }^{*} \mathrm{P}<0.05$, TRN vs. CON. was significantly higher in the TRN than in the CON [9.8\%; $\mathrm{P}<0.05$ (Fig. 1A)]. In contrast, the proportion of the IIb isoform in the TRN was significantly lower than that in the $\operatorname{CON}(13.1 \% ; \mathrm{P}<0.05)$. The proportions of the I and IIa isoforms were similar between the TRN and CON.

In the Wistar rats, the proportion of the IIx isoform in the TRN was significantly higher than that in the $\operatorname{CON}[4.91 \% ; \mathrm{P}<0.05$ (Fig. 1B)]. In contrast, the proportions of the I, IIa, and IIIb isoforms were similar between the TRN and CON.

\section{Protein content}

In the SD rats, the phosphorylation level of p70S6k in the TRN was higher than that in the CON $(\mathrm{P}<0.05)$. The phosphorylation level of Akt and mTOR 


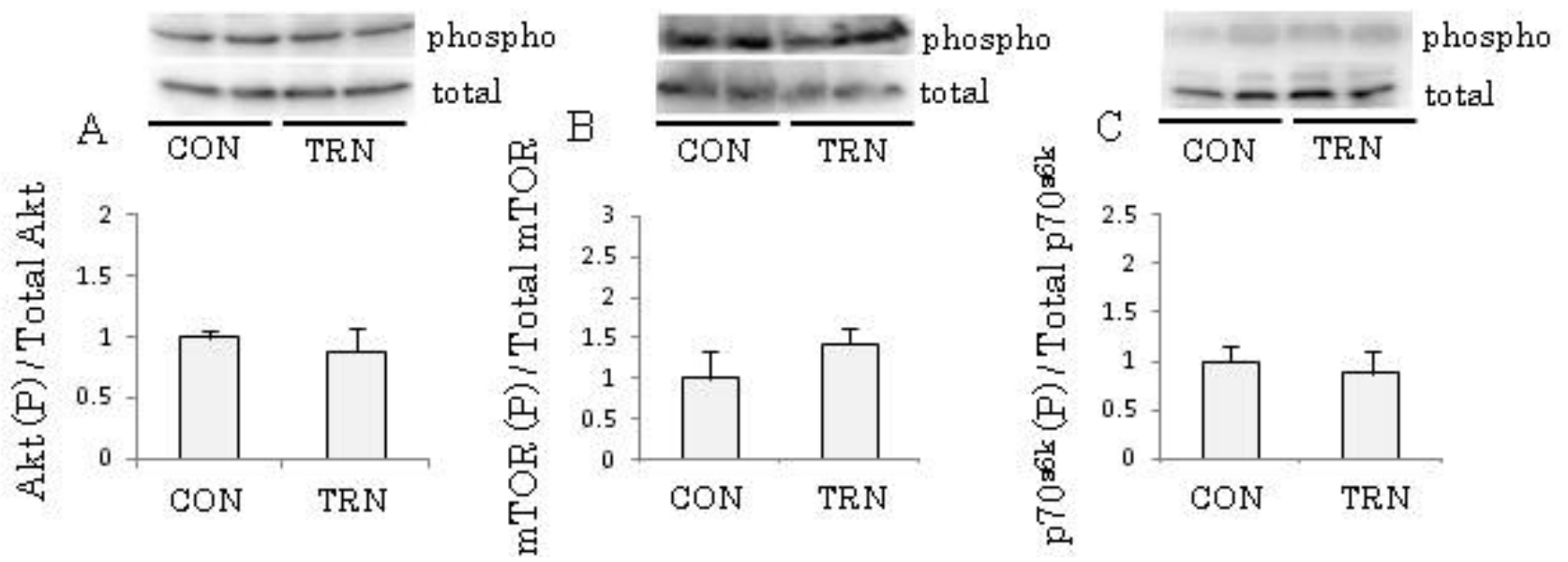

Fig. 4. The ratio of $A k t(P)$ to total $A k t(\mathbf{A})$, the ratio of mTOR $(P)$ to total mTOR (B), and the ratio of p70S6k (P) to total p70S6k (C) in the SD-TRN and -CON determined by western blotting. No significant difference was observed between TRN and CON. Values are expressed as means \pm SE. TRN vs. CON.
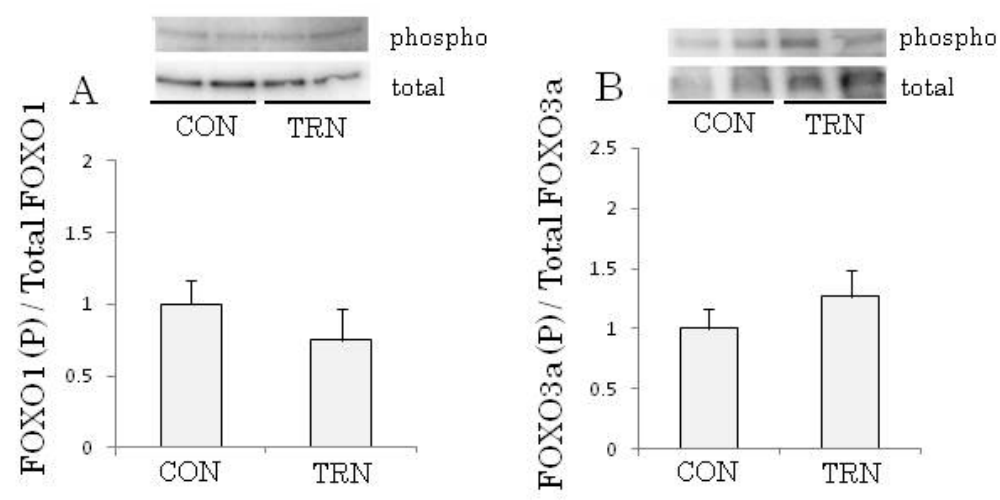

Fig. 5. The ratio of FOXO1 $(P)$ to total FOXO1 (A), the ratio of FOXO3a (P) to total FOXO3a (B), and the levels of MuRF1 (C) and MaFbx/Atrogin-1 (D) in the $\mathrm{W}$-TRN and -CON determined by western blotting. No significant difference was observed between TRN and CON rats. Values are expressed as means $\pm \mathrm{SE}$. TRN vs. CON.

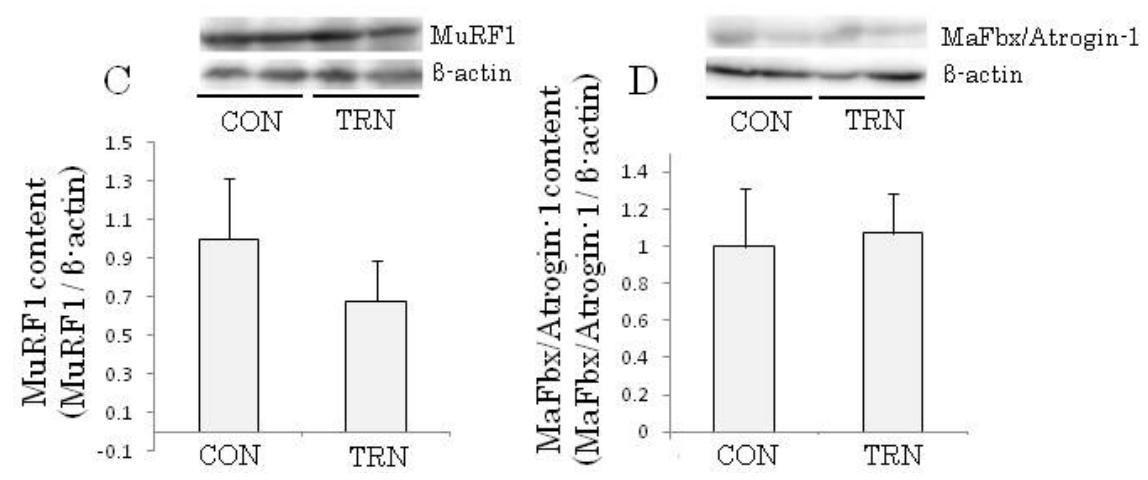

were not significantly different between the TRN and CON. In contrast, in Wistar rats, the phosphorylation level of p70S6k, akt, mTOR in the TRN were not significantly different from those in the CON (Fig. 2).

Phosphorylation level of FOXO3a in the TRN was significantly higher than that in the $\mathrm{SD}(\mathrm{P}<0.05)$. The protein contents of MuRF1 and MaFbx/Atrogin-1 in the TRN were significantly lower than those of the CON $(\mathrm{P}<0.05)$. However, in Wistar rats, phosphorylation level of these protein kinases, and protein contents in the TRN were not significantly different from those in the CON
(Fig. 3).

In Wistar rats, the contents of the examined protein in the W-TRN group were not significantly different from those in the W-CON group (Fig. 4 and 5).

\section{Discussion}

Muscle hypertrophy caused by RT induced through electrical stimulation of the sciatic nerve is a well-established rat model that is used to elucidate intramuscular mechanisms (Haddad et al. 1998, Baar and 
Esser 1999, Adams et al. 2004). The aim of the present study was to determine whether the genetic inter-strain difference in muscle hypertrophy influences the activation of intramuscular signaling cascades associated with muscle size. The primary finding of the present study is that genetic inter-strain differences in RTinduced muscle hypertrophy do indeed exist. Furthermore, this difference appeared to be associated with alterations in intramuscular signaling cascades related to protein metabolism.

In $\mathrm{SD}$ rats, the $\mathrm{LG}$ mass of the TRN was significantly greater $(7.8 \%)$ than that of the CON after 12 sessions of isometric RT. The same tendency was shown in a previous study using a similar RT model (Haddad et al. 1998). They demonstrated that 12 sessions of isometric training increased the $\mathrm{MG}$ mass (13\%) in $\mathrm{SD}$ rats, suggesting that isometric training in the rat model successfully induces muscle hypertrophy in SD rats. Because we electrically stimulated the sciatic nerve, not only the gastrocnemius but also the lower leg muscles were activated. We are convinced that a fixed pedal provides larger mechanical loads to induce hypertrophy of the gastrocnemius, as previously reported by other group (Haddad et al. 1998).

On the other hand, the LG mass of the W-TRN was similar to that of the W-CON. This result indicates that isometric training fails to induce muscle hypertrophy in Wistar rats. In the past, several researchers have reported that there are genetic strain differences between $\mathrm{SD}$ and Wistar rats with respect to muscle fiber type, memory, and behavior (Wyss et al. 2000, Rittenhouse et al. 2002, Novák et al. 2010). To our knowledge, we are the first to show that the muscle anabolic response of SD rats is higher than that of Wistar rats under mechanical stimuli.

Furthermore, genetic strain-dependent differences in muscle fiber type (e.g., in the soleus and extensor digitorum longus) have been demonstrated between SD and Wistar rats (see Novák et al. 2010). Rat muscle fibers are classified into 4 types: type I, type IIa, type IIx, and type IIB; each type has a different contraction speed, fatigue tolerance, metabolism, and muscle fiber size. Since fiber type composition is dependent on metabolic states in skeletal muscle, it is possible that inter-strain differences exist in muscle adaptation to resistance exercise stimulation.

In the present study, the fiber type levels of control LG muscles were similar between SD and Wistar rats. Furthermore, the occurrence rate of type IIx increased significantly in both SD and Wistar rats. It is a well-known fact that high-intensity RT upregulates the fast-to-slow MyHC isoform transition (from type IIb to types IIx and IIa). Haddad et al. (1998) also reported that 12 sessions of RT significantly reduces the type IIb $\mathrm{MyHC}$ ratio and concomitantly increases type IIx MyHC in SD rats. As the muscle weights after RT differed significantly between SD and Wistar rats, it was unexpected that their MyHC isoform compositions and $\mathrm{MyHC}$ isoform transitions were no different. Fiber-typing techniques, such as ATPase staining and immunostaining, would provide further information.

It should be noted that the body weights of the SD and Wistar rats were different. Although the weights of the rats used in this study were almost the same at the time of delivery (ref. Methods), they differed by the end of the RT regimen. Because testosterone, which is a hormone secreted by the testis, elicits protein synthesis, we suspect that this difference in growth influences the response to RT. As the stage of growth influences muscle adaptation (Kumar et al. 2009), in the present study we adjusted the results by age before making comparisons. In the future, we will need to conduct additional studies in fully grown rats.

A number of studies have shown that protein synthesis-related signaling is activated after resistance exercise (Baar and Esser 1999, Nader and Esser 2001, Ochi et al. 2010, Roschel et al. 2011). In the present study, we found that the p70S6k phosphorylation of the $\mathrm{SD}-\mathrm{TRN}$ increased relative to that of the SD-CON $24 \mathrm{~h}$ after the last training session. However, the levels of phosphorylated Akt and mTOR were similar between the SD-TRN and SD-CON. Several researchers have shown that exercise-induced p70S6k activity is maintained for more than $24 \mathrm{~h}$ after exercise (Baar and Esser 1999, Hernandez et al. 2000, Lai et al. 2004). Since Akt and mTOR are upstream molecules of p70S6k, phosphorylated Akt and mTOR might decay earlier than p70S6k. However, Akt, mTOR, and p70S6k phosphorylation did not change in Wistar rats. These results, when combined with those for muscle mass, suggest that muscle hypertrophy is related to molecules involved in protein synthesis.

In the present study, we observed that RT in SD rats that received isometric training decreased MaFbx/Atrogin-1 and MuRF1 protein contents and concomitantly increased FOXO3a phosphorylation. A comparable result that chronic resistance exercise decreases the mRNA expression levels of E3 ligases has 
also been reported (Zanchi et al. 2009). These reports suggest that FOXO proteins and E3 ubiquitin ligases fluctuate similarly-under various mechanical conditions. Moreover, RT reduces protein degradation and causes muscle hypertrophy. However, this tendency was not observed in Wistar rats. Since significant hypertrophy was not observed, it is consistent that protein degradation was not suppressed.

In contrast to SD rats, Wistar rats showed little or no response to RT, suggesting that they are "low responders" to RT. On the other hand, Ochi et al. (2010) successfully induced muscle hypertrophy in Wistar rats after eccentric RT using the same training machine. We speculate that the difference in the anabolic responses of the muscles is due to differences in the stimulation volume and contraction type. We suspect that this variation in the stimulation volume caused the difference in muscle hypertrophy. Further consideration of training volume is necessary to understand the appropriate mechanical stimulus for muscle hypertrophy. Eccentric but not concentric contraction training in humans has been reported to increase the IGF-1 mRNA content (Bamman et al. 2001). Moreover, maximal eccentriccontraction exercise has been confirmed to increase the
p70S6k phosphorylation for over $24 \mathrm{~h}$ (Baar and Esser 1999). In the future, we will apply eccentric and isometric contraction exercises in Wistar rats and compare subsequent molecular events involved in protein synthesis and degradation; this will further clarify the reason for isometric training failing to induce muscle hypertrophy.

In conclusion, 12 sessions of isometric RT induced significant muscle hypertrophy in SD but not Wistar rats. Upregulated protein synthesis and downregulated protein degradation were only observed in $\mathrm{SD}$ rats. These results indicate that the sensitivity to RT is higher in SD rats than in Wistar rats, suggesting the existence of genetic inter-strain differences in muscle adaptation. This phenomenon may be useful for studying individual differences in response to RT.

\section{Conflict of Interest}

There is no conflict of interest.

\section{Acknowledgements}

This work was supported by Japan Society for the Promotion of Science (JSPS) KAKENHI (00307993).

\section{References}

ADAMS GR, CHENG DC, HADDAD F, BALDWIN KM: Skeletal muscle hypertrophy in response to isometric, lengthening, and shortening training bouts of equivalent duration. J Appl Physiol 96: 1613-1618, 2004.

BAAR K, ESSER K: Phosphorylation of p70 (S6k) correlates with increased skeletal muscle mass following resistance exercise. Am J Physiol 276: C120-C127, 1999.

BAMMAN MM, SHIPP JR, JIANG J, GOWER BA, HUNTER GR, GOODMAN A, MCLAFFERTY CL Jr, URBAN RJ: Mechanical load increases muscle IGF-I and androgen receptor mRNA concentrations in humans. Am $J$ Physiol 280: E383-E390, 2001.

BIOLO G, MAGGI SP, WILLIAMS BD, TIPTON KD, WOLFE RR: Increased rates of muscle protein turnover and amino acid transport after resistance exercise in humans. Am J Physiol 268: E514-E520, 1995.

BODINE SC, STITT TN, GONZALEZ M, KLINE WO, STOVER GL, BAUERLEIN R, ZLOTCHENKO E, SCRIMGEOUR A, LAWRENCE JC, GLASS DJ, YANCOPOULOS GD: Akt/mTOR pathway is a crucial regulator of skeletal muscle hypertrophy and can prevent muscle atrophy in vivo. Nat Cell Biol 3: 1014-1019, 2001.

DREYER HC, FUJITA S, CADENAS JG, CHINKES DL, VOLPI E, RASMUSSEN BB: Resistance exercise increases AMPK activity and reduces 4E-BP1 phosphorylation and protein synthesis in human skeletal muscle. $J$ Physiol 576: 613-624, 2006.

DREYER HC, DRUMMOND MJ, PENNINGS B, FUJITA S, GLYNN EL, CHINKES DL, DHANANI S, VOLPI E, RASMUSSEN BB: Leucine-enriched essential amino acid and carbohydrate ingestion following resistance exercise enhances mTOR signaling and protein synthesis in human muscle. Am J Physiol 294: E392-E400, 2008.

HADDAD F, QIN AX, ZENG M, MCCUE SA, BALDWIN KM: Effects of isometric training on skeletal myosin heavy chain expression. J Appl Physiol 84: 2036-2041, 1998. 
HADDAD F, ADAMS GR, BODELL PW, BALDWIN KM: Isometric resistance exercise fails to counteract skeletal muscle atrophy processes during the initial stages of unloading. J Appl Physiol 100: 433-441, 2006.

HERNANDEZ JM, FEDELE MJ, FARRELL PA: Time course evaluation of protein synthesis and glucose uptake after acute resistance exercise in rats, $J$ Appl Physiol 88: 1142-1149, 2000.

HORNBERGER TA, HUNTER RB, KANDARIAN SC, ESSER KA: Regulation of translation factors during hindlimb unloading and denervation of skeletal muscle in rats. Am J Physiol 281: C179-C187, 2001.

KUMAR V, SELBY A, RANKIN D, PATEL R, ATHERTON P, HILDEBRANDT W, WILLIAMS J, SMITH K, SEYNNES O, HISCOCK N, RENNIE MJ: Age-related differences in the dose-response relationship of muscle protein synthesis to resistance exercise in young and old men. $J$ Physiol 587: 211-217, 2009.

LAI KM, GONZALEZ M, POUEYMIROU WT, KLINE WO, NA E, ZLOTCHENKO E, STITT TN, ECONOMIDES AN, YANCOPOULOS GD, GLASS DJ: Conditional activation of Akt in adult skeletal muscle induces rapid hypertrophy. Mol Cell Biol 24: 9295-9304, 2004.

LEWIS DM, LEVI AJ, BROOKSBY P, JONES JV: A faster twitch contraction of soleus in the spontaneously hypertensive rat is partly due to changed fibre type composition. Exp Physiol 79: 377-386, 1994.

LOUIS E, RAUE U, YANG Y, JEMIOLO B, TRAPPE S: Time course of proteolytic, cytokine, and myostatin gene expression after acute exercise in human skeletal muscle. J Appl Physiol 103: 1744-1751, 2007.

MIZUNOYA W, WAKAMATSU J, TATSUMI R, IKEUCHI Y: Protocol for high-resolution separation of rodent myosin heavy chain isoforms in a mini-gel electrophoresis system. Anal Biochem 377: 111-113, 2008.

NADER GA, ESSER KA: Intracellular signaling specificity in skeletal muscle in response to different modes of exercise. J Appl Physiol 90: 1936-1942, 2001.

NOVÁK P, ZACHAŘOVÁ G, SOUKUP T: Individual, age and sex differences in fiber type composition of slow and fast muscles of adult Lewis rats: comparison with other rat strains. Physiol Res 59: 783-801, 2010.

OCHI E, ISHII N, NAKAZATO K: Time course change of IGF1 / Akt / mTOR / p70S6k pathway activation in rat gastrocnemius muscle during repeated bouts of eccentric exercise. J Sports Sci Med 9: 170-175, 2010.

RITTENHOUSE PA, LÓPEZ-RUBALCAVA C, STANWOOD GD, LUCKI I: Amplified behavioral and endocrine responses to forced swim stress in the Wistar-Kyoto rat. Psychoneuroendocrinology 27: 303-318, 2002.

ROSCHEL H, UGRINOWISTCH C, BARROSO R, BATISTA MA, SOUZA EO, AOKI MS, SIQUEIRA-FILHO MA, ZANUTO R, CARVALHO CR, NEVES M, MELLO MT, TRICOLI V: Effect of eccentric exercise velocity on akt/mtor/p70(s6k) signaling in human skeletal muscle. Appl Physiol Nutr Metab 36: 283-290, 2011.

SANDRI M, SANDRI C, GILBERT A, SKURK C, CALABRIA E, PICARD A, WALSH K, SCHIAFFINO S, LECKER SH, GOLDBERG AL: FoxO transcription factors induce the atrophy-related ubiquitin ligase atrogin1 and cause skeletal muscle atrophy. Cell 117: 399-412, 2004.

STITT TN, DRUJAN D, CLARKE BA, PANARO F, TIMOFEYVA Y, KLINE WO, GONZALEZ M, YANCOPOULOS GD, GLASS DJ: The IGF-1/PI3K/Akt pathway prevents expression of muscle atrophyinduced ubiquitin ligases by inhibiting FoxO transcription factors. Mol Cell 14: 395-403, 2004.

WYSS JM, CHAMBLESS BD, KADISH I, VAN GROEN T: Age-related decline in water maze learning and memory in rats: strain differences. Neurobiol Aging 21: 671-681, 2000.

ZANCHI NE, DE SIQUEIRA FILHO MA, LIRA FS, ROSA JC, YAMASHITA AS, DE OLIVEIRA CARVALHO CR, SEELAENDER M, LANCHA-JR AH: Chronic resistance training decreases MuRF-1 and Atrogin-1 gene expression but does not modify Akt, GSK-3beta and p70S6k levels in rats. Eur J Appl Physiol 106: 415-423, 2009. 